\title{
EDITORIAL
}

\section{THE VITREOUS}

The Vitreous Symposium at the 1992 Annual College Congress provided an opportunity to review current knowledge of the biochemistry, anatomy, pathology and physics of the vitreous. In this issue of Eye, five papers derived from that symposium serve to keep ophthalmologists abreast of developments in the basic sciences applied to this part of the eye.

The last few years have seen major advances in our understanding of the biochemistry of the mammalian vitreous which, happily for ophthalmology, is an extracellular matrix (ECM) of great interest to biochemists since the way collagen molecules blend with their interfibrillar ground substance largely determines the properties of tissues. The work of Balazs characterised the vitreous as a meshwork of randomly organised, unbranching type II collagen fibrils dispersed in ground substance made up of coiled hyaluronan (HA) molecules. ${ }^{1}$ More recently, further collagens and glycosaminoglycans (GAGs) have been discovered in the vitreous, most notably the demonstration that $15 \%$ of total vitreous collagen is type IX collagen; ${ }^{2}$ latterly isolation of the intact form of type IX collagen from bovine vitreous has shown it to be a proteoglycan (PG), with a short covalently linked chondroitin sulphate/dermatan sulphate (CS/DS) GAG chain. ${ }^{3}$ Electron microscopy after rotary shadowing reveals the type IX collagen/PG extensively coating or 'decorating' the surface of type II fibrils as a series of projecting 'struts' within the loose HA network. ${ }^{4,5}$ Furthermore, certain GAGs found in vitreous (including HA and some forms of chondroitin sulphate) have been shown to associate as flat tape-like structures of helical dimers formed either between themselves or each other (homo- and hetero-duplexes). ${ }^{6}$ In this issue, John Scott from the University of Manchester, using his electron microscopic staining technique employing Cupromeronic blue at critical electrolyte concentration, demonstrates the periodic or ladder-like sulphated GAG linkages between type II collagen fibrils (possibly as homoduplexes via the CS/DS GAG chain of type IX collagen/PG) and goes on to synthesise current concepts of the supramolecular organisation of the vitreous which he likens to "very dilute corneal stroma'. ${ }^{7}$ He proposes that while forming an extensive three-dimensional anastomosing network of laterally associated molecules, ${ }^{5} \mathrm{HA}$ might even interact with the type II collagen fibrils via the type IX collagen/PG (albeit conceding that neither this interaction nor the short CS/DS chains are currently evident by rotary shadowing). Based on these advances, it could be that certain enzymes (such as chondroitin ABC lyase) will in due course transfer from the biochemist's bench to our ophthalmic surgical armamentarium to enable selective enzymic or 'chemical' vitrectomy.

The vitreous gel is not, of course, homogeneous and further work is required to determine the biochemical basis of biomicroscopically apparent interfaces in, and condensations of, the vitreous gel such as the posterior hyaloid 'membrane', together with the means by which the closely packed fibrils of the vitreous cortex attach to (and detach from) the inner limiting lamina of the retina. How a type II collagen disorder such as Stickler's syndrome results in an 'optically empty' gel and posterior hyaloid anomalies should then become clearer. In this issue, Jerry Sebag from the University of Southern California reviews the 'membranellar' structure of the vitreous, firstly in its historical context and then with particular emphasis on the pathological and surgical implications of splitting of the posterior hyaloid membrane ('vitreoschisis'). ${ }^{8}$ This process is particularly relevant to macular hole formation, hypocellular epiretinal membranes and proliferative diabetic retinopathy. Non-mechanical approaches to the separation of gel and retina are eagerly awaited.

The vitreous ECM is thought to have a crucial role in preretinal neovascularisation, absence of cortical gel precluding epiretinal neovascularisation except for tiny 'abortive' outgrowths and new vessels confined to the optic disc. ${ }^{9,10}$ In keeping with Michaelson's hypothesis, ${ }^{11}$ a large number of growth factors have been successfully harvested from the vitreous of diabetic patients, though the notion of a 'bespoke' vitreous angiogenic factor has receded. However, the extent to which angiogenesis depends on the local oxygen milieu rather than the presence of such molecular modulators is currently under debate; ${ }^{12}$ in this issue, Mike Boulton and colleagues from the University of Manchester show that growth modulators may even arise from the neovascular tissue itself. They also attempt to reconcile opposing views on the pathophysiology of preretinal neovascularisation by demonstrating that the local oxygen tension has a profound influence on the response of retinal microvascular cells to growth modulators and also on the production of such factors in vitro ${ }^{13}$ This interplay of matrix, modu- 


\section{EDITORIAL}

lators and milieu probably determines the varied clinical manifestations of neovascularisation following retinal capillary non-perfusion.

The production of other ECM components within epiretinal membranes is considered by Paul Hiscott and colleagues from the Institute of Ophthalmology in this issue. ${ }^{14}$ Machemer, while promulgating the cellular basis of epiretinal membrane formation in the 1970s, nevertheless emphasised the role of type I collagen in consolidating any cell-mediated membrane contraction. ${ }^{15}$ More recent studies have suggested a prior role for other ECM component molecules such as fibronectin and vitronectin. ${ }^{16,17}$ This latest contribution from Ian Grierson's group concerns another ECM glycoprotein - thrombospondin - which may be involved in the assembly of the ECM in epiretinal membranes by binding to fibronectin and modulating the membrane ECM via the integrin family of cell-surface receptors. ${ }^{14}$ A clearer biochemical picture of the development of epiretinal membranes is thus emerging together with the basis of their tractional effects on the underlying retina.

Finally the physical consequences of epiretinal membrane formation, whether by mediating traction or during surgical manipulation, are reported in this issue by Ian Jones and colleagues from the University of Cambridge. ${ }^{18}$ The distortion, stretching and elevation of the retina seen clinically (e.g. in proliferative diabetic retinopathy) are consequent upon the operation of stresses and forces which can be quantified. ${ }^{19}$ Removal of epiretinal membranes often leads to apparent restoration of structure which suggests retinal 'elasticity', but sometimes the retina is irreversibly deformed or ectopic (from disruption of elastic structures). Otherwise, the retina becomes completely disrupted or torn which, although a manifestation of traction, is by the same token evidence of relief of traction. Using in vitro techniques, the 'physics' of these vitreoretinal inter-relationships have been further explained especially in relation to the role of heavy liquids in epiretinal membrane dissection. ${ }^{18,19}$ It is to be hoped that such physics-based studies will help ophthalmologists to 'optimise' their vitreoretinal surgical techniques, at least while we await further intraoperative assistance from the biochemists.

DAVID MCLEOD

\section{REFERENCES}

1. Balazs EA: Molecular morphology of the vitreous body. In: Smelser GK, ed. The Structure of the Eye. New York and London: Academic Press, 1961: 293-310.

2. Ayad S, Weiss JB: A new look at vitreous humour collagen. Biochem J 1984, 218: 835-40.

3. Bishop P, McLeod D, Ayad S: Extraction and characterisation of the intact form of bovine vitreous type IX collagen. Biochem Biophys Res Commun 1992, 185: 392-7.

4. Ren ZX, Brewton RG, Mayne R: An analysis by rotary shadowing of the structure of the mammalian vitreous humour and zonular apparatus. J Struct Biol 1991, 106: 57-63.

5. Brewton RG and Mayne R: Mammalian vitreous humour contains networks of hyaluronan molecules: electron microscopic analysis using the hyaluronan-binding region (G1) of aggrecan and link protein. Exp Cell Res 1992, 198: 237-49.

6. Scott JE: Supramolecular organisation of extracellular matrix glycosaminoglycans in vitro and in the tissies. FASEB J 1992, 6: $2639-45$

7. Scott JE: The chemical morphology of the vitreous. Eye 1992, 6: 553-555.

8. Sebag J: Anatomy and pathology of the vitreo-retinal interface. Eye 1992, 6: 541-552.

9. Wong HC, Sehmi K, McLeod D: Abortive neovascular outgrowths discovered during vitrectomy for diabetic vitreous haemorrhage. Graefes Arch Clin Exp Ophthalmol 1989, 227:237-40.

10. Hiscott P, Rosen R, Cooling RJ, Garner A: The pathology of abortive neovascular outgrowths. Graefes Arch Clin Exp Ophthalmol 1992, 230: 531-6.

11. Michaelson IC: The mode of development of the vascular system of the retina, with some observations for its significance for certain retinal diseases. Trans Ophthalmol Soc UK 1948, 68: 137-86.

12. Stefansson E, Wilson CA, Schoen T, Kuwabara T: Experimental ischaemia induces cell mitosis in the adult rat retina. Invest Ophthalmol Vis Sci 1988, 29: 1050-5.

13. Boulton ME, Patel B, Khaliq A, Moriarty P, Jarvis-Evans J, McLeod D: Modulators and milieu in preretinal neovascularisation. Eye 1992, 6: 560-565.

14. Hiscott P, Larkin G, Robey HL, Orr G, Grierson I: Thrombospondin as a component of the extracellular matrix of epiretinal membranes: comparisons with cellular fibronectin. Eye 1992, 6: 566-569.

15. Machemer R: Pathogenesis and classification of massive periretinal proliferation. Br J Ophthalmol 1978, 62: 737-47.

16. Hiscott P, Grierson I, McLeod D: Natural history of fibrocellular epiretinal membranes: a quantitative, autoradiographic and immunohistochemical study. Br J Ophthalmol 1985, 69: 810-23.

17. Weller M, Weidemann P, Bresgen M, Heimann K: Vitronectin and proliferative intraocular disorders: a co-localisation study of the serum spreading factor, vitronectin and fibronectin in traction membranes from patients with proliferative vitreoretinopathy. Int Ophthalmol 1991, 15: 93-101.

18. Jones IL, Warner M, Stevens JD: Mathematical modelling of the elastic properties of retina: a determination of Young's modulus. Eye 1992, 6: 556-559.

19. Stevens JD, Jones IL, Warner M, Lavin MJ, Leaver PK: Mathematical modelling of retinal tear formation: implications for the use of heavy liquids. Eye 1992, 6: 69-74. 